\title{
Chirped pulses sum frequency generation for deep-UV picosecond pulse shaping
}

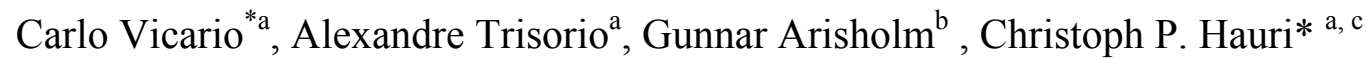 \\ ${ }^{a}$ Paul Scherrer Institute, 5232 Villigen PSI, Switzerland; \\ ${ }^{\mathrm{b}}$ FFI (Norwegian Defence Research Establishment), P O Box 25, NO-2027 Kjeller, Norway; \\ ${ }^{\mathrm{c}}$ Ecole Polytechnique Federale de Lausanne, 1015 Lausanne, Switzerland
}

\begin{abstract}
We show by experiments and simulations that properly chirped laser pulses enable efficient and broadband sum frequency generation in nonlinear crystals. We achieved high energy, picosecond deep-UV pulses with spectral width one order of magnitude greater than the acceptance bandwidth of the nonlinear interaction. The broad spectrum supports shaping of ps flat-top deep-UV pulses with short rise- and fall-time, which are optimal for driving high brightness photocathode electron guns.
\end{abstract}

Keywords: Harmonic generation, pulse shaping, deep-UV pulse

\section{INTRODUCTION}

The present paper demonstrates high-energy, temporally shaped laser pulses to drive a high-brightness photocathode electron gun. Flat-top-like picosecond pulses at $260-280 \mathrm{~nm}$ with less than $1 \mathrm{ps}$ rise and fall time are required to generate initial photoelectron beams for accelerators that drive X-ray Free Electron Lasers (FEL)[1]. This temporal profile can increase the electron beam brightness, which is the key parameter to enhance the FEL performance [2]. Due to the low quantum efficiency of the present gun photocathodes, pulses on the order of hundred $\mu \mathrm{J}$ are needed [3].

In our system, the deep-UV (DUV) shaped pulses are generated by sum frequency generation (SFG) between the fundamental and the second harmonic $(\omega+2 \omega=3 \omega)$ of a Ti:sapphire (Ti:Sa) laser. The temporal shaping in the IR takes advantage of the broad spectrum of the Ti:Sa laser. For a flat-top pulse the minimum attainable rise time depends on the spectral bandwidth available [4]. However, the conversion of broadband pulses is difficult because of the limited acceptance bandwidth of both the second harmonic generation (SHG) and SFG stages. Broadband conversion with ordinary phase matching would require thin nonlinear crystals, associated to low nonlinear process efficiency. Femtosecond pulses with high peak power can be employed to increase the harmonic efficiency. However, the laser damage threshold is reduced for fs pulses, and this restricts the maximum pulse energy on a non-linear medium of limited size. Furthermore, when the goal is ps flat-top pulses, additional temporal stretching and shaping would be required, and such temporal manipulations are rather inefficient in the DUV [5]. For this reason, it is desirable to do the shaping in the IR, but this requires that the conversion process does not alter the pulse shape.

In this paper, we present experiments and simulations where, for the first time, chirp-matched SFG [6] is paired with an IR pulse shaper to generate and shape high-energy DUV pulses. We show that the broad spectra obtained permit flat-top pulses with short rise- and fall-time. Chirp-matching (CM) SFG is achieved by tailoring the chirp of the generating pulses so that perfect phase matching is realized for each input frequency pair. If the temporal rate of chirp is small, the frequency change within the temporal walk-off time will be negligible. In this case the spectral width is not restricted to the acceptance bandwidth of the crystal, and longer crystals can be used to increase the conversion efficiency. For type I

*carlo.vicario@psi.ch; phone+41 (0)563103162; http://www.psi.ch/index_e.shtml

*christoph.hauri@psi.ch; phone+41 (0)563104197; http://www.psi.ch/index_e.shtml

Ultrafast Phenomena and Nanophotonics XVI,

Edited by Markus Betz, Abdulhakem Y. Elezzabi, Jin-Joo Song, Kong-Thon Tsen,

Proc. of SPIE Vol. 8260, 82600J - (c) 2012 SPIE - CCC code: 0277-786X/12/\$18 - doi: 10.1117/12.915596

Proc. of SPIE Vol. 8260 82600J-1 
phase matching in $\mathrm{BBO}$, the $\mathrm{CM}$ condition is satisfied for the $\mathrm{SFG}$ process $800+400 \rightarrow 266 \mathrm{~nm}$ if the group velocity dispersion of the fundamental, $\Phi_{1}{ }^{\prime}$, and of the second harmonic $\Phi_{2}{ }^{\prime}$ fulfills the relation: $\Phi_{1}{ }^{\prime \prime} / \Phi_{2}{ }^{\prime}=-1.8[6]$. In general, when the chirps are adjusted to match the above condition, the spectral phase and intensity of the output pulse are simply related to the corresponding properties of the input pulses. In this case IR shapers can be effectively employed for highresolution control of temporal intensity in the DUV.

\section{EXPERIMENTAL SETUP}

Figure 1 shows the experimental setup. The source is a TW-class, Ti:Sa laser system equipped with Dazzler and Mazzler acousto optical filters (Fastlite) for the control of the spectral phase and the bandwidth. The amplifier delivers near infrared pulses at $100 \mathrm{~Hz}$ with variable bandwidth between 15 and $130 \mathrm{~nm}$ FWHM [7].

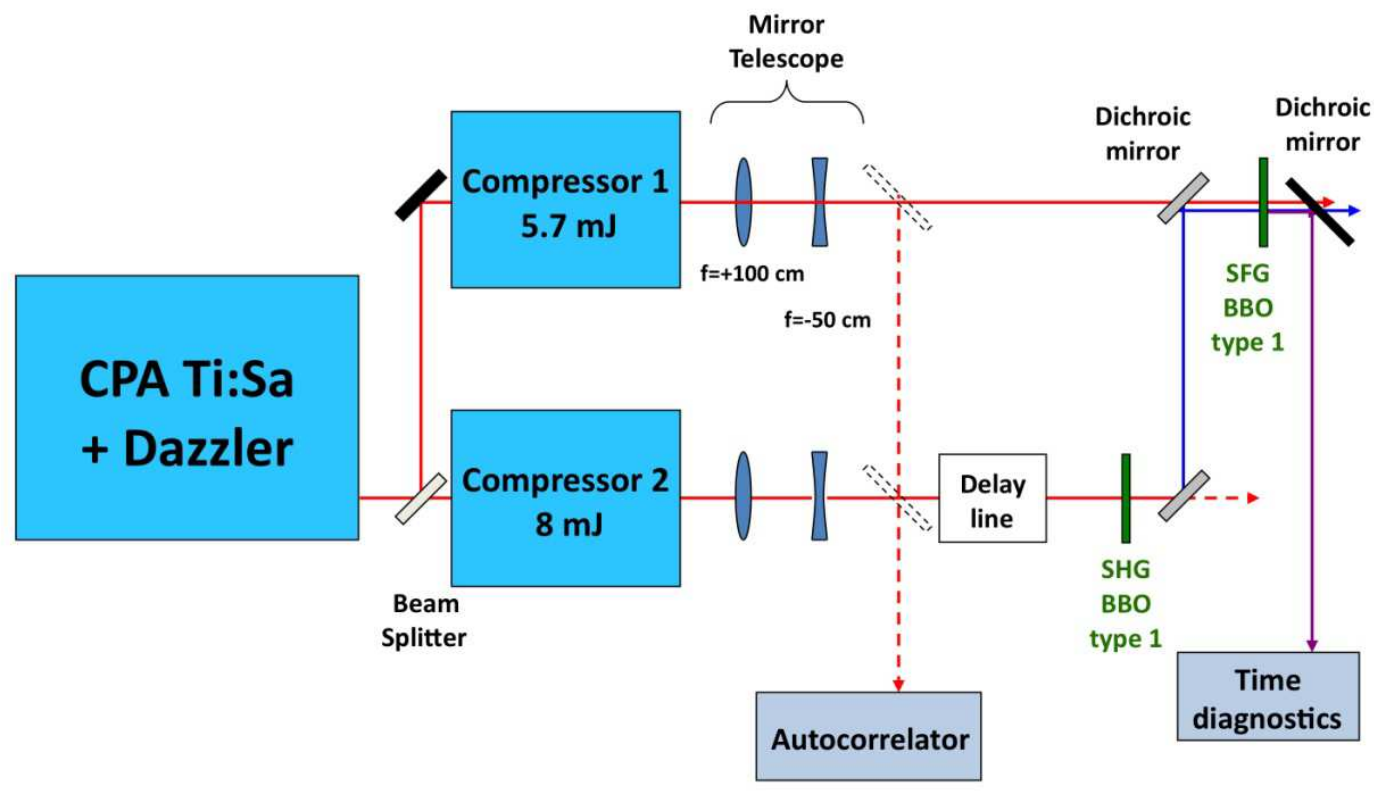

Figure 1 Layout of the experimental setup.

The amplifier is pumped with 7 diode-pumped Nd:YAG units. This pumping scheme permits remarkable energy stability of $0.4 \% \mathrm{rms}$ and $2.7 \%$ ptp measured over 500 shots. The amplified pulses are split and separately compressed. The two output pulses can be independently chirped and delayed to realize the CM condition. For both arms, the transverse profile is approximately Gaussian with FWHM diameter $D$ of $10 \mathrm{~mm}$. The energy is unequally divided into the two beams $(8$ and $5.7 \mathrm{~mJ}$ ) to provide more energy for the SHG out of the compressor 2. Both the SHG and SFG stages are realized with BBO crystals with type I phase matching. The SHG, which does not take advantage of the chirp matching, must have short crystals $(0.1$ or $0.2 \mathrm{~mm})$ to support the necessary bandwidth. For the SFG a crystal of $0.8 \mathrm{~mm}$ was used. The input IR pulse length is retrieved with a commercial auto-correlator (PulseCheck, APE GmbH). The output pulse at $267 \mathrm{~nm}$ is measured by cross-correlation in the same instrument where the residual IR pulse from the SHG is recompressed down to 160 fs FWHM and then used as probe.

\section{SIMULATIONS AND EXPERIMENTAL RESULTS}

In a first experiment, we show that CM SFG allows high energy and a DUV spectrum much broader than the acceptance bandwidth of the non-linear interaction. For this purpose, CM SFG and unmatched SFG are compared under conditions 
where only the chirp of one of the input pulses changes and other parameters are fixed. Then we present the SFG sensitivity with respect to the chirp ratio and the relative delay between the input pulses. Finally we show experimentally and theoretically that the CM SFG paired with spectral shaping of the IR pulse can be used for DUV temporal shaping. The resulting top-hat ps pulses are highly suitable for driving the electron gun in an X-ray FEL application.

The experimental results in the temporal and spectral domains are compared with numerical simulations. The calculations are based on coupled equations for the amplitudes in the spatial and temporal frequency domains. Only the spectral amplitudes are restricted to vary slowly compared to the optical wavelength, so there is no restriction on the pulse length. Dispersion and birefringence of the nonlinear medium are included exactly $[8,9]$. For the simulations, the $800 \mathrm{~nm}$ input pulse was calculated by taking the measured spectrum and applying a linear chirp to achieve the measured pulse duration. The beam transverse profile was approximated by a Gaussian with $10 \mathrm{~mm}$ diameter. The output energy from the SHG simulation was somewhat lower than the measured energy, possibly because of the approximate beam profile or an inaccurate value for the nonlinear coefficient, so the SHG energy was scaled to the measured value before input to the SFG simulation.

CU SFG

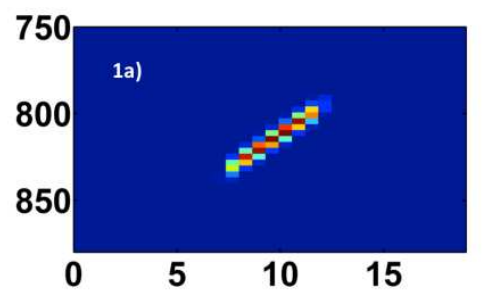

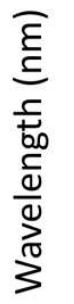
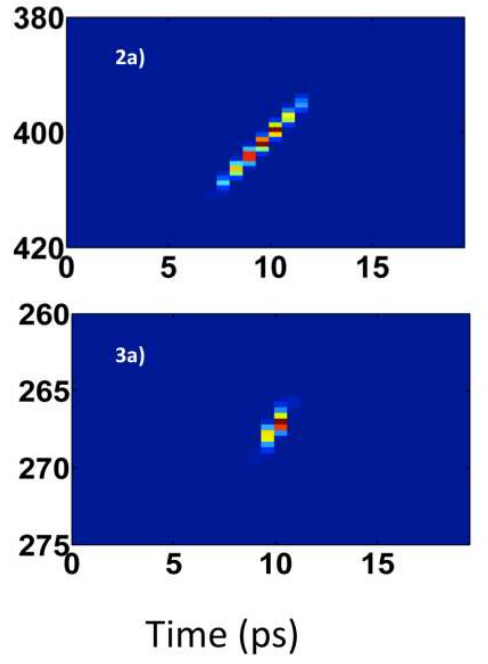

\section{SFG}
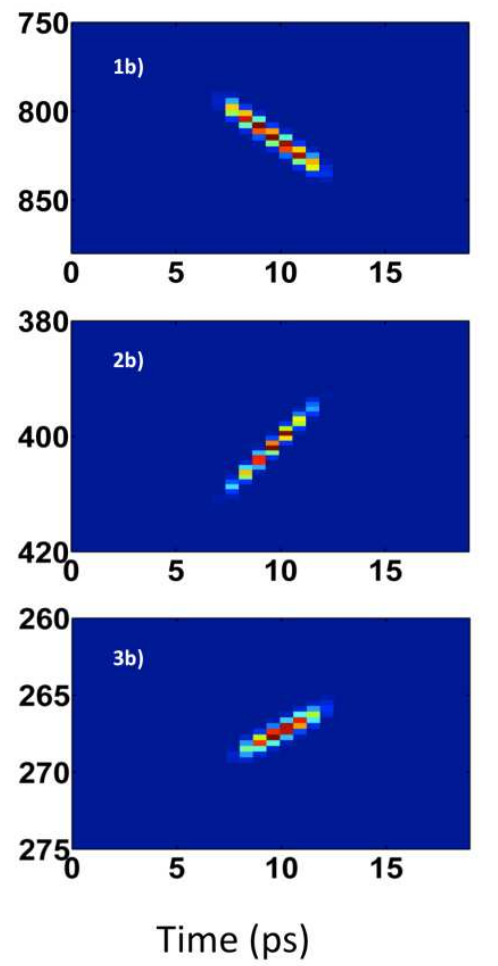

Figure 2 Simulated spectrograms for the fundamental, second harmonic and DUV pulses in case of a) chirp unmatched and b) chirp matched sum frequency generation.

In figure 2 simulations for $\mathrm{CM}$ and chirp unmatched (CU) SFG are reported. The images represent the spectrograms for the fundamental, second harmonic and the generated DUV pulses. These calculations successfully reproduce the experimental DUV spectra that are shown in figure 3 (blue curves). For consistent comparison between the two conditions we kept the input peak power constant. The only difference between the two cases is that the chirp of the IR signal is inverted. For both simulations and experiments, the fundamental and second harmonic spectra were respectively 35 and $13 \mathrm{~nm}$ wide (FWHM) and the input pulses were 5.1 and $4.9 \mathrm{ps}$ long. As reported in figure $3 \mathrm{~b}$ ) the bandwidth of the UV pulse in the CM case is $2.5 \mathrm{~nm}$ FWHM, five times larger than in the CU case 3a). The energy is also significantly improved: the measured energy is $220 \mathrm{uJ}$ for CM case and only $35 \mathrm{uJ}$ for CU. It is worth mentioning that in the $\mathrm{CM}$ case the depletion of the second harmonic beam was $65 \%$. 

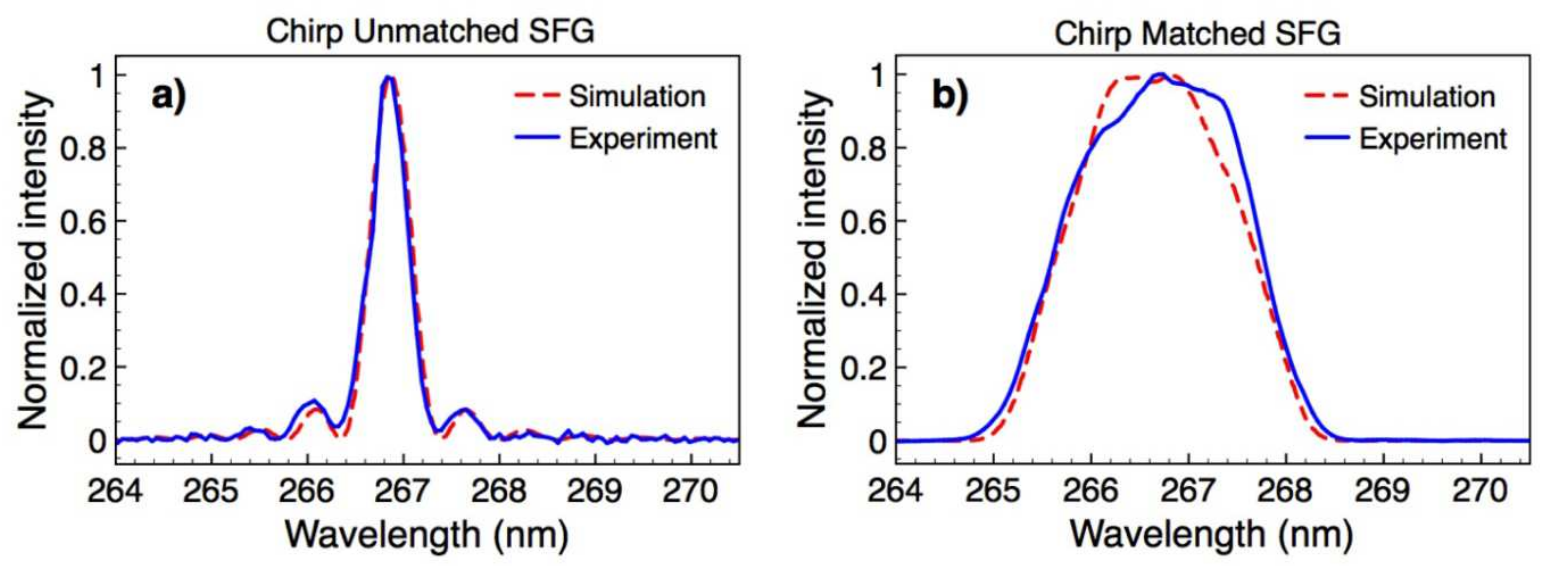

Figure 3 Output experimental (blue) and simulated (red) spectra for a) CU and b) CM SFG.

By reducing the IR beam diameter $D$ down to $6 \mathrm{~mm}$ FWHM, we achieved second harmonic energy $E_{2}=1.1 \mathrm{~mJ}$, DUV energy of $E_{3}=0.85 \mathrm{~mJ}$ (stability of $2 \% \mathrm{rms}$ ) and $1 \mathrm{~nm}$ bandwidth. The recorded energy is comparable with the value obtained in our lab with $50 \mathrm{fs}$ harmonic generation and $0.35 \mathrm{~mm}$ thick SFG crystal. However, in the latter case additional lossy manipulation is required to produce the ps target pulses.

Using a broader input spectrum it is possible to extend the bandwidth of the output signal even further. The DUV spectra shown in figure 4 are obtained with IR spectra of $70 \mathrm{~nm}$ and $0.1 \mathrm{~mm}$ SHG crystal. The CM DUV spectrum has a width of more than $4 \mathrm{~nm}$ (FWHM), which is nine times greater than for CU SFG, and the energy was $50 \mu \mathrm{J}$. The generated bandwidth can support ultrashort pulses.

The delay $\Delta t$ between the fundamental and the second harmonic strongly impacts the value $E_{3}$ and the output bandwidth $\Lambda_{0}$. When $\Delta t$ is varied in the range $+/-400 \mathrm{fs}$ around the optimal overlap, the UV spectrum becomes asymmetric and narrow. The relative change in spectral width can be approximated as: $\left(\Lambda(\Delta \mathrm{t})-\Lambda_{\max }\right) / \Lambda_{\max }=-4.4 \cdot 10^{-6}\left[\mathrm{fs}^{-2}\right] \cdot \Delta \mathrm{t}^{2}$.

The DUV bandwidth as function of the relative delay is shown in figure 5a). The output DUV energy varies with similar law: $\left(\mathrm{E}_{3}(\Delta \mathrm{t})-\mathrm{E}_{3 \max }\right) / \mathrm{E}_{3 \max }=-4.2 \cdot 10^{-6}\left[\mathrm{fs}^{-2}\right] \cdot \Delta \mathrm{t}^{2}$. For $\Delta t=300 f s$, a modest value respect to the $5 \mathrm{ps}$ pulse duration, the energy and the bandwidth diminish by $39 \%$ and $37 \%$ respectively.

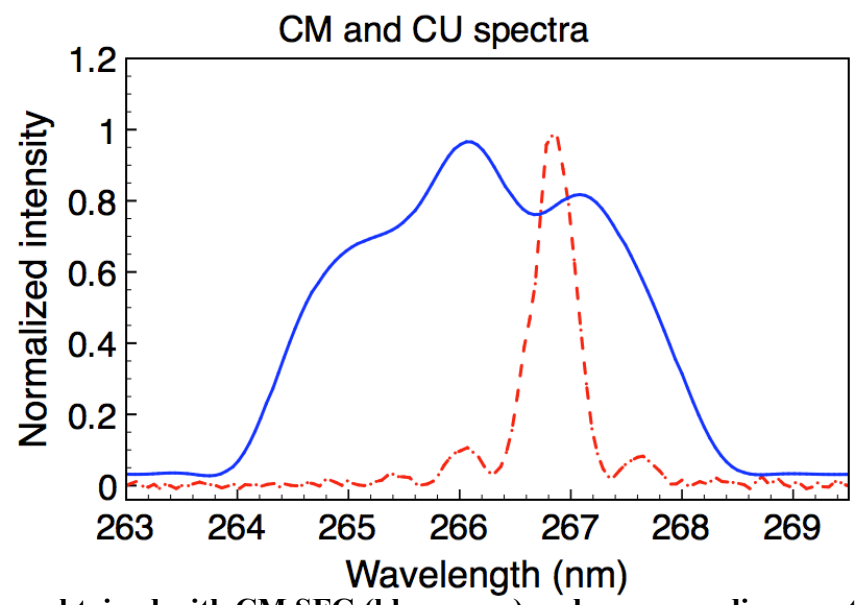

Figure 4 Ultrabroad spectrum obtained with CM SFG (blue curve) and corresponding spectrum for CU SFG (red dashed curve). 
The SFG is also very sensitive to the deviation from the optimal chirp ratio $\Phi_{1}{ }^{\prime \prime} / \Phi_{2}{ }^{\prime \prime}: 8 \%$ relative variation from the optimum reduces the SFG bandwidth by $20 \%$. The output spectral width as function of the deviation from the optimal chirp ration of -1.8 , obtained for $\Phi_{1}{ }^{\prime \prime}=+4.3 \cdot 10^{4}$ and $/ \Phi_{2}{ }^{\prime \prime}=-2.4 \cdot 10^{4}$ is reported in figure $5 \mathrm{~b}$ ).
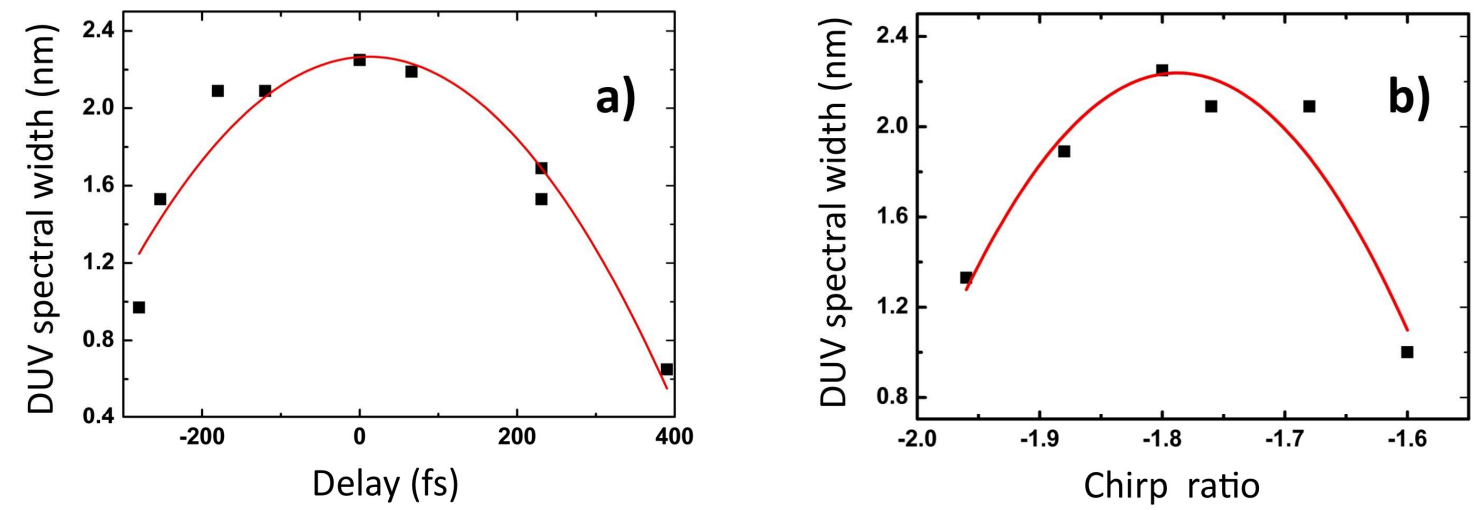

Figure 5 DUV spectral widths a) as function of relative delay between the generating pulses, and b) as function of chirp ratio between the two beams.

\section{FLAT TOP PULSES BY CHIRP MATCHED SFG}

To produce the flat-top profile required for the electron gun, we combined IR pulse shaping and CM-SFG. For this experiment the Dazzler was programmed to perform spectral intensity shaping in the IR. With this scheme we demonstrated that high-resolution temporal shaping in the DUV is feasible.

At the CM condition the SFG pulse becomes linearly chirped with temporal chirp rate equal to the sum of the ones of the input pulses. When the pulses are long enough, as in our application, there is a direct correspondence between the SFG spectrum and the temporal pulse profile. Therefore, the UV pulse shape can be controlled by spectral shaping of the input spectra prior to the SFG. The IR spectrum is shaped directly, but the blue spectrum is distorted by the SHG process. However, it has a steep edge on one side, and by suitable timing of the input pulses to the SFG we were able to obtain a nearly flat-top DUV spectrum with steep edges on both sides.

In figure 6 the experimental results of the flat-top DUV generation are presented (blue curves) and compared to the simulations (red plots). To obtain the flat-top DUV pulse, the Dazzler was programmed to shape a $30 \mathrm{~nm}$ wide squarelike IR spectrum with sharp edges (10-90\%) of less than $2 \mathrm{~nm}$ (figure 6a). The IR pulse lengths out of the compressors were 6.1 and $5.7 \mathrm{ps} \mathrm{FWHM.} \mathrm{To} \mathrm{satisfy} \mathrm{the} \mathrm{CM} \mathrm{condition} \mathrm{in} \mathrm{the} \mathrm{SFG,} \mathrm{the} \mathrm{chirp} \mathrm{of} \mathrm{the} \mathrm{fundamental} \mathrm{wavelength} \mathrm{and} \mathrm{the}$ second harmonic were set respectively at $-7.5 \cdot 10^{4} \mathrm{fs}^{2}$ and $+4.05 \cdot 10^{4} \mathrm{fs}^{2}$. For the SHG and the SFG process, $0.1 \mathrm{~mm}$ and $0.8 \mathrm{~mm}$ BBO crystals were used. The second harmonic spectrum is asymmetric with one sharp edge, fig 6b). As shown in figure 6c), the obtained DUV spectrum is flat top with full bandwidth of $2.5 \mathrm{~nm}$. Figure 6d) shows the DUV pulse measured with the cross-correlator and the simulated temporal profile for the fundamental, second harmonic and DUV pulses. Since the DUV pulse is driven by the product of the input pulses, the long trailing edge of the blue pulse is effectively masked by the steep edge of the IR pulse, and the resulting DUV pulse is has a flat top. The shaped pulse is 4.5 ps FWHM and the rise and fall times are shorter than 0.8 ps. The modulations at the plateau are $10 \% \mathrm{rms}$ and $39 \%$ ptp. The obtained pulse shape satisfies the requirements of the high brightness laser driven electron sources. Moreover the achieved energy of $200 \mathrm{uJ}$ permits the generation of sufficient charge for standard metal photocathodes for X-ray FEL. 

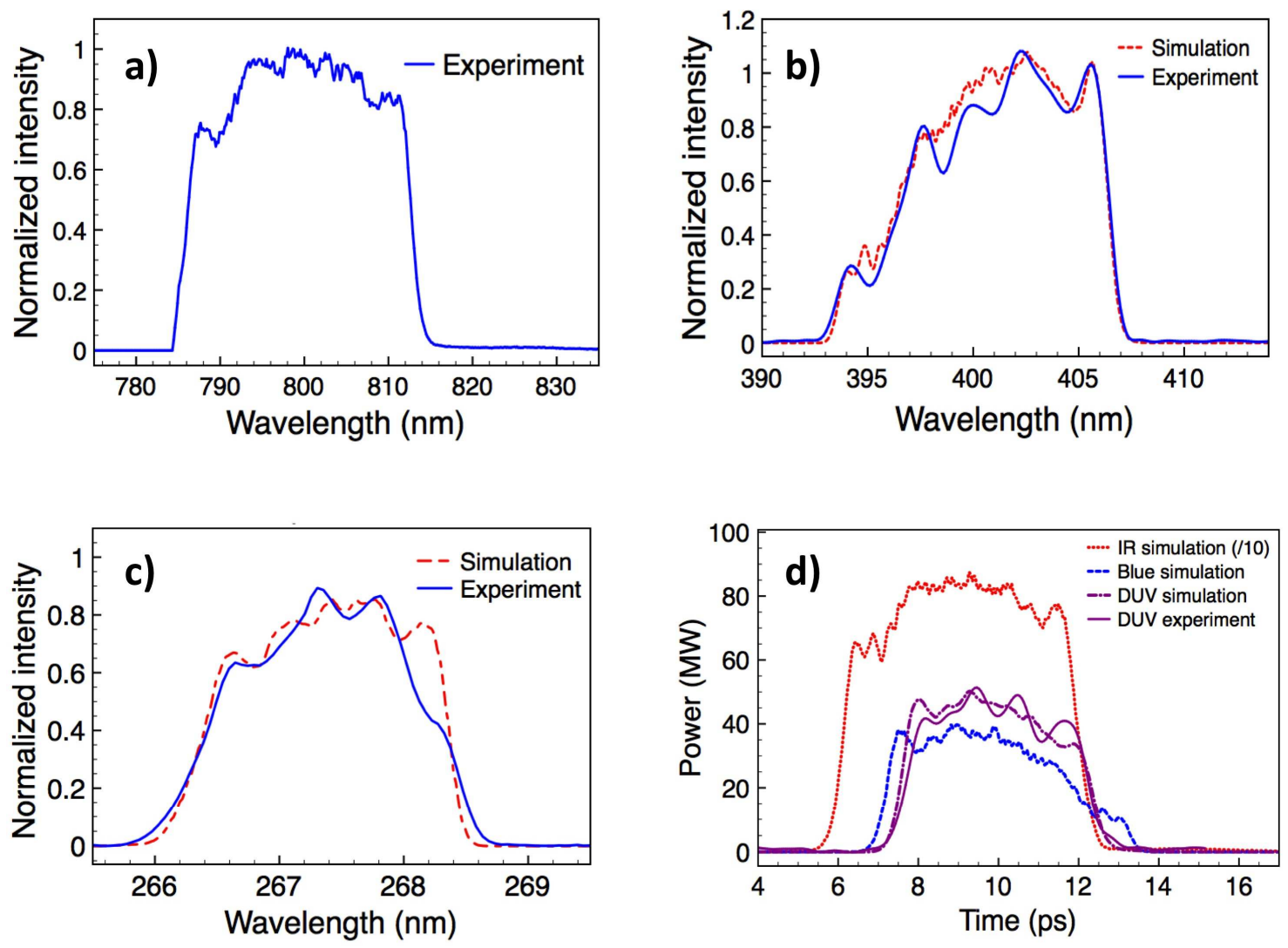

Figure 6 Spectral intensity of the (a) fundamental and (b) second harmonic. (c) DUV spectrum and (d) pulse shapes. The experimental results (blue curves) are compared with simulations (red curves)

\section{CONCLUSION}

In conclusion, with the CM SFG technique, we achieved a DUV spectral width of more than $4 \mathrm{~nm}$, nine times larger than the acceptance bandwidth of the mixing process. We also demonstrated high DUV pulse energy for broadband picosecond harmonic generation. The technique is suitable for efficient generation of high-resolution ps flat-top pulses in the DUV, where temporal shaping is still challenging. The experimental results in the spectral and temporal domain have been accurately reproduced by numerical simulation. In the present setup, the maximum energy and spectral width obtainable in the DUV are limited by the SHG.

The authors are grateful to N. Forget and P. Tournois from Fastlite for discussions. This work was partially supported by the Swiss National Science Foundation grant PP00P2_128493 (CPH).

\section{REFERENCES}

[1] B. D. Patterson et al., "Coherent science at the SwissFEL x-ray laser", New Journal of Physics 12, 035012 (2010).

[2] J. Yang at al., "Low-emittance electron-beam generation with laser pulse shaping in photocathode radiofrequency gun", J. Appl. Phys. 92, 1608 (2002).

[3] C. P. Hauri, R. Ganter, F. Le Pimpec, A. Trisorio, C. Ruchert and H. H. Braun, "Intrinsic Emittance Reduction of an Electron Beam from Metal Photocathodes", Phys. Rev. Lett. 104, 234802 (2010). 
[4] S.Cialdi, C. Vicario, M. Petrarca P. Musumeci "Simple scheme for UV time pulse shaping", Appl. Opt. 46, 4959 (2007).

[5] A. Trisorio, C. Ruchert and C. P. Hauri "Direct shaping of picosecond high energy deep ultraviolet pulses", Appl. Phys B, 105, 255 (2011).

[6] K. Osvay and I. N. Ross "Broadband sum-frequency generation by chirp-assisted group-velocity matching", J. Opt. Soc. Am. B, 13, 1431 (1996).

[7] A. Trisorio, P. M. Paul, F. Ple, C Ruchert, C, Vicario and C. P. Hauri, "Ultrabroadband TW-class Ti:sapphire laser system with adjustable central wavelength, bandwidth and multi-color operation", Opt. Expr., 19, 20128, (2011).

[8] G. Arisholm "General numerical methods for simulating second order nonlinear interactions in birefringent media," J. Opt. Soc. Am. B 14, 2543 (1997).

[9] G. Arisholm "Quantum noise initiation and macroscopic fluctuations in optical parametric oscillators" J.Opt. Soc. Am. B, 16, 117 (1999). 